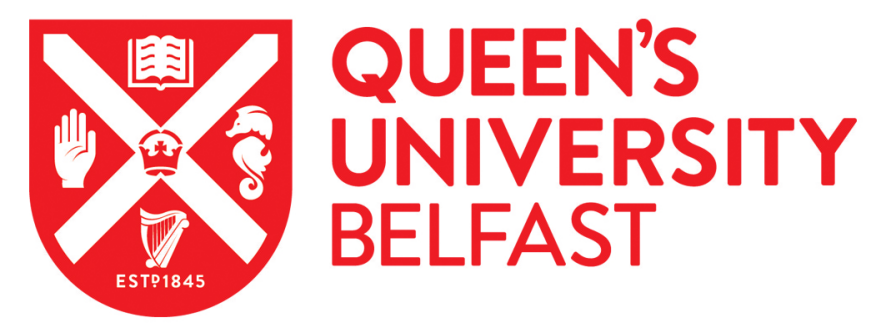

\title{
Uncovering Predictability in the Evolution of the WTI Oil Futures Curve
}

Kearney, F., \& Shang, H. L. (2020). Uncovering Predictability in the Evolution of the WTI Oil Futures Curve. European Financial Management, 26(1), 238-257. https://doi.org/10.1111/eufm.12212

\author{
Published in: \\ European Financial Management
}

Document Version:

Peer reviewed version

Queen's University Belfast - Research Portal:

Link to publication record in Queen's University Belfast Research Portal

Publisher rights

(c) 2019 John Wiley \& Sons, Ltd. This work is made available online in accordance with the publisher's policies. Please refer to any applicable terms of use of the publisher.

\section{General rights}

Copyright for the publications made accessible via the Queen's University Belfast Research Portal is retained by the author(s) and / or other copyright owners and it is a condition of accessing these publications that users recognise and abide by the legal requirements associated with these rights.

Take down policy

The Research Portal is Queen's institutional repository that provides access to Queen's research output. Every effort has been made to ensure that content in the Research Portal does not infringe any person's rights, or applicable UK laws. If you discover content in the Research Portal that you believe breaches copyright or violates any law, please contact openaccess@qub.ac.uk. 


\title{
Uncovering predictability in the evolution of the WTI oil futures curve
}

\author{
Fearghal Kearney* \\ Queen's Management School \\ Queen's University Belfast
}

Han Lin Shang ${ }^{\dagger}$

Research School of Finance, Actuarial Studies and Statistics Australian National University

December 2, 2018

\begin{abstract}
Accurately forecasting the price of oil, the world's most actively traded commodity, is of great importance to both academics and practitioners. We contribute by proposing a functional time series based method to model and forecast oil futures. Our approach boasts a number of theoretical and practical advantages including effectively exploiting underlying process dynamics missed by classical discrete approaches. We evaluate the finite-sample performance against established benchmarks using a model confidence set test. A realistic out-of-sample exercise provides strong support for the adoption of our approach with it residing in the superior set of models in all considered instances.
\end{abstract}

Keywords: crude oil, forecasting, functional time series, futures contracts, futures markets

*Corresponding author: Queen's Management School, Queen's University Belfast, BT9 5EE, UK. Telephone: +44 28 9097 4795. Email: f.kearney@qub.ac.uk

${ }^{\dagger}$ Research School of Finance, Actuarial Studies and Statistics, Level 4, Building 26C, Australian National University, Kingsley Street, Canberra ACT 2601, Australia. Telephone: +61 26125 0535. Email: hanlin.shang@anu.edu.au 


\section{Introduction}

Traditionally, statistical frameworks adopted in both the academic literature and practice address the modelling of low-frequency regularly spaced datasets. However, the recent availability of higher-resolution time series brings with it unique challenges and opportunities (Engle 2000). Functional Time Series (FTS) is a convenient modelling and forecasting framework boasting the ability to tackle such problems. Tsay (2016) demonstrates that FTS analysis is widely applicable to many areas of big data analytics and business statistics. We take up this mantle and propose a novel exponential smoothing state space FTS framework to address a critical problem in empirical finance, namely the modelling and forecasting of crude oil futures prices.

Alquist et al. (2013) outline three critical areas for which reliable forecasts of oil prices are essential. First, airlines, automobile firms, utilities and even homeowners make pricing, consumption and investment decisions based on forecasts of oil prices. Second, oil price forecasts are a key component of central bank macroeconomic projections and market practitioners' risk assessments, with more accurate forecasts potentially improving policy responses and risk management strategies. Third, oil price forecasts inform energy usage and carbon emission projections with regulatory policy implications such as fuel excise adjustments and climate change interventions. More specifically, in this paper, we focus on oil futures prices. In general, commodity futures are far more actively traded than their related spot contracts (see, e.g., Ng and Pirrong 1996). Outlining the importance of futures markets, Laws and Thompson (2004) explicitly identify four functions they serve: efficient price discovery, resource allocation, risk management and financing. Furthermore, energy futures price forecasts can accurately inform the pricing of related securities and projects (Kyriakou et al. 2016).

The use of such financial derivatives in Europe has been widely documented (see, e.g., Carroll et al. 2017). More specifically for oil derivatives, Brent crude was traditionally regarded as the leading indicator of prices within Europe. However, as the United States (US) recently repealed its 40-year-old ban on exporting WTI (West Texas Intermediate) crude, forecasting WTI crude oil futures has become a vital issue for European audiences. Since the repeal of this ban, there has been a surge in WTI futures trading. ${ }^{1}$ This phenomenon has led to a trend of increased trading volumes for WTI oil futures coupled with reduced trading volumes for the traditionally European Brent oil futures. ${ }^{2}$

Furthermore, recent production of oil in the European North Sea has seen a structural decline at a time when US WTI crude has observed record production levels. This has led to reports that the firm who set the price of Brent, S\&P Global Platts, is now considering using WTI prices to

\footnotetext{
${ }^{1}$ Reuters, “U.S. oil output surges but growth likely to moderate in 2019”, 1 November 2018.

${ }^{2}$ Financial Times, “US oil futures market overtakes London", 17 September 2017.
} 
calculate its latest European index. ${ }^{3}$ These factors have culminated in WTI arguably becoming the price discovery leader in global crude oil markets and overtaking Brent as the leading oil benchmark in Europe. ${ }^{4}$

Two main classes of models are outlined in the commodity futures modelling literature: discrete latent factor and fundamental macroeconomic-based models. Latent factor approaches to model commodity futures curves include those presented by Casassus and Collin-Dufresne (2005), Chantziara and Skiadopoulos (2008), Trolle and Schwartz (2009) and Casassus et al. (2013), with a separate strand of literature concluding that fundamentals are important in explaining energy prices (see, e.g., Kilian 2009, Singleton 2013, Andreasson et al. 2016, Basak and Pavlova 2016). Based on this literature we adopt the fundamental commodity futures forecasting framework of Andreasson et al. (2016), as well as the principal component approach of Chantziara and Skiadopoulos (2008) as two benchmarks for our proposed FTS model. This aligns with the approach taken by Cummins et al. (2016) who adopt both frameworks and find no discernible difference between the performance of the latent and fundamental models.

To the best of our knowledge, this paper constitutes the first application of FTS methods to describe, model and forecast commodity futures curves. ${ }^{5}$ In the FTS domain, the item of interest is a set of curves, shapes, objects or, more broadly, functional observations. Because trading is focused on specific segments of the oil futures curve, quotes are only observable at discrete expiry points. However, the novel approach that we propose (described in Section 3) seeks to uncover the true underlying curve dynamics that is often missed by traditional techniques. This unearthed underlying process is subsequently projected forward using an exponential smoothing model that has widespread application in business forecasting (see, e.g., Hyndman et al. 2008, Taylor 2010). In an exponential smoothing setting, forecasts are weighted combinations of past observations-in our case, the functional principal component decomposition of daily oil futures curves. The idea is that the most recent observations inform the forecast most heavily, with weights decreasing exponentially for more distant past observations. We evaluate our forecasting models using the data snooping bias-free model confidence set (MCS) procedure of Hansen et al. (2011), as also employed by Neumann and Skiadopoulos (2013) and Baruník and Malinská (2016). Through this, we ascertain that the FTS approach is superior relative to the benchmark models.

In addition to producing more accurate one-day ahead out-of-sample oil price forecasts, the

\footnotetext{
${ }^{3}$ Bloomberg, "U.S. Oil Could Soon Help Set the Brent Crude Price", 6 July 2018

${ }^{4}$ CME Group, "WTI Entering a Renaissance as a Global Benchmark", 17 July 2018.

${ }^{5}$ A number of previous empirical studies has benefitted from being cast in a functional data environment. Prominent examples include credit card transactions (Laukaitis 2008), online auction price dynamics (Wang et al. 2008) and electricity price curves (Chen and Li 2017). More specifically within financial markets, applications span equity index volatility (Müller et al. 2011), stock returns (Horváth et al. 2014, Horváth and Rice 2015, Kokoszka et al. 2015, 2018, Zhang 2016), yield curves (Kowal et al. 2017) and option volatility (Liu et al. 2016, Kearney et al. 2015, 2018).
} 
use of an FTS framework boasts several practical and conceptual advantages. First, the approach effectively captures serial dependence, a prominent attribute of daily oil futures curves. Secondly, it facilitates the modelling of data observed at a small number of unequally spaced points, as well as those with possible non-negligible measurement error, which are common in business statistics (see, e.g., Kowal et al. 2018). In our specific context, we follow Cummins et al. (2016) in focusing on the most actively traded futures contract expiries (CL1-CL9, CL12, and CL18), resulting in unequally spaced data points along the futures curve, which our FTS framework models effectively. Third, the approach facilitates the pricing of non-standard expiry OTC (over-the-counter) contracts, as the output is a complete forecasted futures curve across an essentially infinite number of expiries. Finally, and more practically, given that it relies on latent factors, our approach circumvents the need to a priori identify fundamental factors that model oil futures prices, thus mitigating the risk of omitted variable bias. The paper continues as follows: Section 2 describes the oil futures dataset, Section 3 outlines our proposed FTS methodology and forecasting evaluation framework, Section 4 presents in- and out-of-sample results and Section 5 concludes the paper.

\section{Oil Futures Data}

Crude oil is the world's most heavily traded commodity futures contract (see, e.g., Sévi 2014). More specifically, Bernardi et al. (2018) outline that WTI crude oil has the highest weighting of all futures across the three leading commodity indices compiled by Bloomberg, UBS and Dow Jones. Each WTI futures contract is written on 1,000 barrels of crude oil, whereby a price is agreed today for oil to be delivered and paid for on a specific date in the future. On any given day, a large number of contracts is traded with expiration dates in consecutive months. Generic series can be constructed by rolling from one contract to another, with CL1 representing the oil futures contract with the shortest date to expiry, CL2 the second shortest and so forth. Because of lower trading intensity, liquidity levels are reduced for certain non-standard maturities observed along the futures curve. To alleviate any such concerns, we concentrate only on the most actively traded generic monthly futures in line with Cummins et al. (2016). The futures we use are CL1-CL9, CL12 and CL18 obtained from the CME Group. The CME Group is the world's largest marketplace for buying and selling futures contracts. It consists of four separate exchanges: the Chicago Mercantile Exchange, Chicago Board of Trade, New York Mercantile Exchange and Commodity Exchange. Our sample is at a daily frequency from January 2009 to December 2015. To construct the fundamental factor model, daily VIX quotes are downloaded from the Chicago Board of Options Exchange and S\&P 500 (Standard and Poor's 500 index) levels retrieved from Yahoo! Finance, with the Trade Weighted US Dollar Index (USD) and US Economic Policy Uncertainty Index (EcPol) both being obtained from FRED (Federal Reserve Economic Data). Descriptive statistics for our dataset are given in 


\begin{tabular}{lrrrrrrr}
\hline & Mean & Std. Dev. & Median & Minimum & Maximum & Skewness & Kurtosis \\
\hline CL1 & 81.51 & 19.99 & 86.72 & 33.98 & 113.93 & -0.70 & -0.65 \\
CL2 & 82.07 & 19.48 & 87.41 & 35.78 & 114.43 & -0.70 & -0.66 \\
CL3 & 82.50 & 19.00 & 87.94 & 36.79 & 114.71 & -0.71 & -0.65 \\
CL4 & 82.81 & 18.59 & 88.37 & 37.60 & 114.83 & -0.73 & -0.62 \\
CL5 & 83.05 & 18.20 & 88.77 & 38.45 & 114.82 & -0.74 & -0.59 \\
CL6 & 83.21 & 17.83 & 89.10 & 39.07 & 114.78 & -0.75 & -0.55 \\
CL7 & 83.31 & 17.50 & 89.36 & 39.68 & 114.73 & -0.76 & -0.52 \\
CL8 & 83.39 & 17.18 & 89.52 & 40.24 & 114.58 & -0.77 & -0.48 \\
CL9 & 83.44 & 16.88 & 89.56 & 40.88 & 114.38 & -0.78 & -0.44 \\
CL10 & 83.51 & 16.05 & 89.34 & 42.28 & 113.65 & -0.80 & -0.33 \\
CL11 & 83.32 & 14.59 & 88.21 & 44.41 & 111.71 & -0.83 & -0.15 \\
VIX & 20.17 & 8.04 & 17.56 & 10.32 & 56.65 & 1.59 & 2.32 \\
S\&P 500 & $1,481.51$ & 390.40 & $1,385.14$ & 676.53 & $2,130.82$ & 0.18 & -1.19 \\
USD & 77.55 & 6.40 & 75.91 & 68.02 & 94.97 & 1.19 & 0.47 \\
EcPol & 116.10 & 67.15 & 100.85 & 3.32 & 548.95 & 1.51 & 3.61 \\
\hline & & & & & & & \\
\hline
\end{tabular}

Note: This table reports the descriptive statistics of CME Group's historical crude oil continuous futures prices. CL1 represent the next consecutive month's futures contract, CL2 is the following month's futures contract and so forth. The sample period is January 2009-December 2015.

Table 1.

Figure 1 presents plots of the relationship between oil futures prices on selected days in 2009 and 2013. In general, the spread between short- and long-expiry futures can be either positive or negative, with the relationship dependent on underlying market forces. On 30 January 2009 (Figure 1a) longer expiries are more expensive than shorter-expiry contracts, which is known as a state of contango. In Figure 1b, the opposite effect is seen, with longer-expiry futures being cheaper, which is known as backwardation. Our flexible FTS approach effectively characterises both states. Furthermore, Figure 2 presents the evolution of prices for short ( 1 month), medium (6 months) and long expiry (18 months) futures throughout our sample period. It can be seen that the price spread is not constant over time, with Schwartz and Smith (2000) previously establishing that such differences between short- and long-maturity contracts provide substantial information about short-term price variations. We next detail our proposed FTS framework to forecast this oil dataset. 


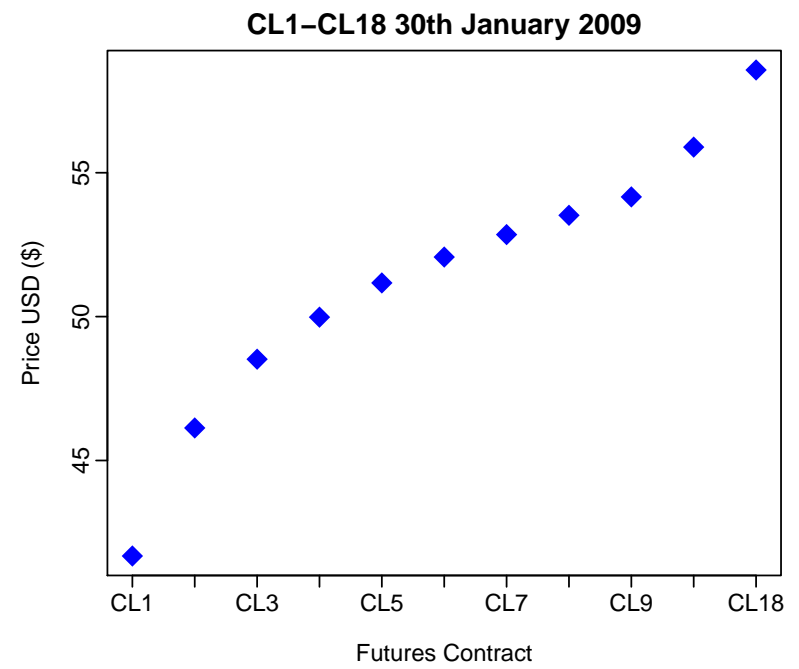

(a) Panel A

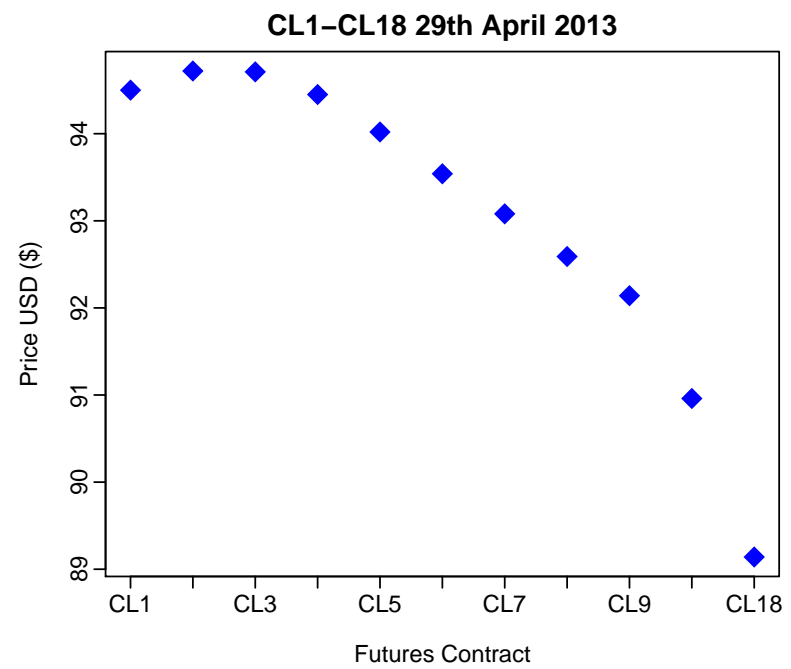

(b) Panel B

Figure 1: The crude oil continuous futures price for each of 11 different expiries on the 30 January 2009 (Panel A) and 29 April 2013 (Panel B)

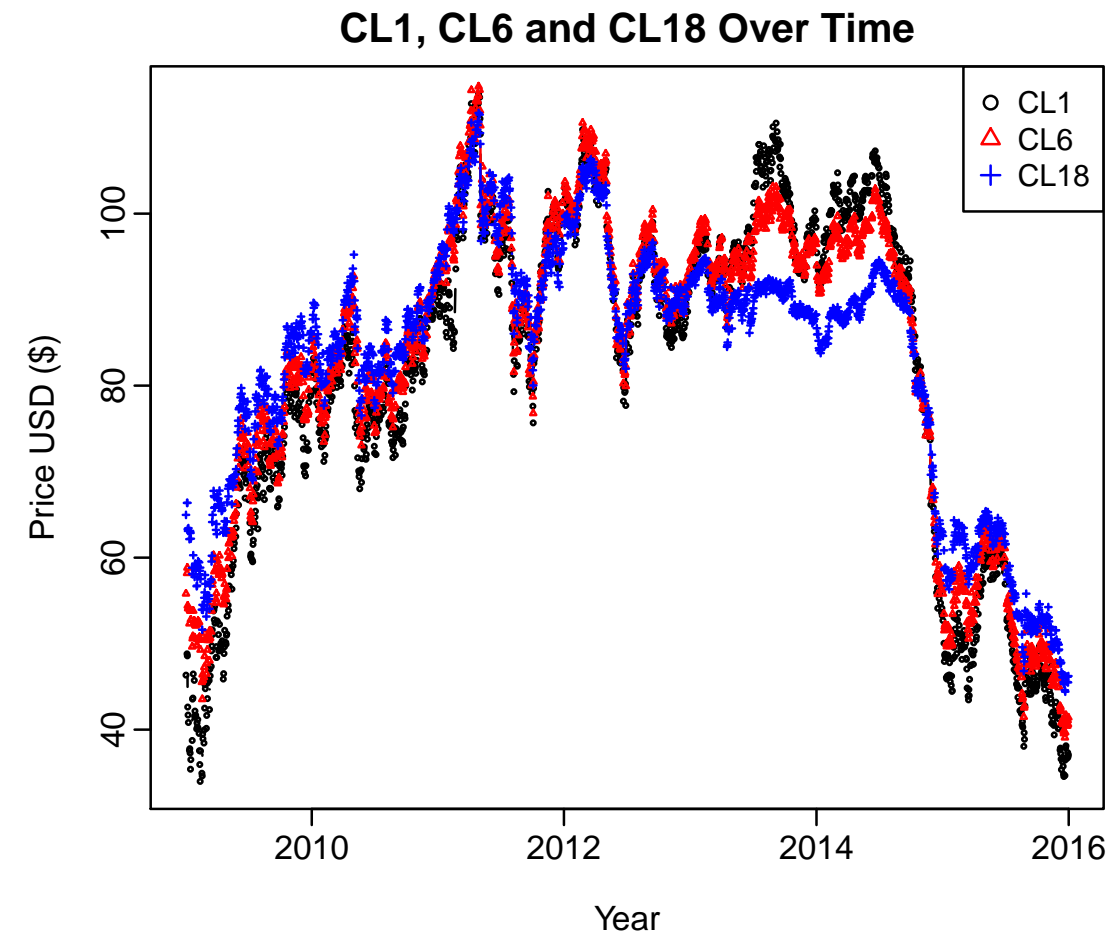

Figure 2: Evolution of three crude oil continuous futures prices over January 2009-December 2015 period; shortterm, medium-term and long-term expiries are represented by CL1, CL6 and CL18, respectively 


\section{Methodology}

\subsection{Exponential Smoothing Functional Time Series Forecasts}

We adopt an FTS approach to first model and subsequently forecast the relationship between the prices associated with oil futures contracts of different expiries. To model this futures curve, we first let $\left(\mathcal{X}_{t}, t \in \mathbb{Z}\right)$ be an arbitrary FTS defined on a common probability space $(\Omega, A, P)$. In our case $\mathcal{X}_{t}$ represents the futures curve on each day $i$, with the continuum $\theta$ given in terms of the futures contract expiry, CL1, CL2, etc. ${ }^{6}$ Each function is a square integrable function satisfying $\left\|\mathcal{X}_{t}\right\|^{2}=\int_{\mathcal{I}} \mathcal{X}_{t}^{2}(\theta) d \theta<\infty .{ }^{7}$ The notation $\mathcal{X} \in \mathcal{L}_{\mathcal{H}}^{p}(\Omega, A, P)$ is used to indicate that $\mathrm{E}\left(\|\mathcal{X}\|^{p}\right)<\infty$ for some $p>0$. For instance, when $p=1, \mathcal{X}(\theta)$ has the mean curve $\mu(\theta)=\mathrm{E}[\mathcal{X}(\theta)]$; when $p=2$, the non-negative definite covariance function is given by

$$
c_{\mathcal{X}}(\theta, \omega)=\operatorname{Cov}[\mathcal{X}(\theta), \mathcal{X}(\omega)]=\mathrm{E}\{[\mathcal{X}(\theta)-\mu(\theta)][\mathcal{X}(\omega)-\mu(\omega)]\},
$$

for all $\theta, \omega \in \mathcal{I}$. The covariance function in (1) allows the covariance operator of $\mathcal{X}$ to be defined as:

$$
\mathcal{K}_{\mathcal{X}}(\phi)(\omega)=\int_{\mathcal{I}} c_{\mathcal{X}}(\theta, \omega) \phi(\theta) d \theta
$$

There then exists an orthonormal sequence $\left(\phi_{k}\right)$ of continuous functions and a non-increasing sequence $\left(\lambda_{k}\right)$ of positive numbers, such that:

$$
c_{\mathcal{X}}(\theta, \omega)=\sum_{k=1}^{\infty} \lambda_{k} \phi_{k}(\theta) \phi_{k}(\omega), \quad \theta, \omega \in \mathcal{I}
$$

Therefore, a Karhunen-Loève expansion of such a stochastic process $\mathcal{X}(\theta)$ can be expressed as:

$$
\begin{aligned}
\mathcal{X}(\theta) & =\mu(\theta)+\sum_{k=1}^{\infty} \beta_{k} \phi_{k}(\theta) \\
& =\mu(\theta)+\sum_{k=1}^{K} \beta_{k} \phi_{k}(\theta)+e(\theta),
\end{aligned}
$$

where scores $\beta_{k}$ are given by the projection of $\mathcal{X}(\theta)-\mu(\theta)$ in the direction of the $k^{\text {th }}$ eigenfunction $\phi_{k}$; that is, $\beta_{k}=\left\langle\mathcal{X}(\theta)-\mu(\theta), \phi_{k}(\theta)\right\rangle ; e(\theta)$ denotes the model truncation error function with a mean of zero and a finite variance; and $K<n$ is the number of retained components. Equation (2) facilitates dimension reduction as the first $K$ terms often provide a good approximation to the

\footnotetext{
${ }^{6}$ In this paper we use the generic rolling contracts, CL1, CL2, etc., for consistency with prior studies including those that propose our adopted benchmark models. However, the FTS framework we propose could also be modified to handle each futures contract's precise number of days to expiry.

${ }^{7}$ It is assumed that the observations $\mathcal{X}_{t}$ are elements of the Hilbert space $\mathcal{H}=\mathcal{L}^{2}(\mathcal{I})$ equipped with the inner product $\langle w, v\rangle=\int_{\mathcal{I}} w(\theta) v(\theta) d \theta$, where $\theta$ represents a continuum and $\mathcal{I} \subset R$ denotes a function support range and $R$ denotes the real line.
} 
infinite sums, and thus the information inherent in $\mathcal{X}(\theta)$ can be adequately summarised by the K-dimensional vector $\left(\boldsymbol{\beta}_{1}, \cdots, \boldsymbol{\beta}_{K}\right)$.

There are at least four common approaches to determine our selection of $K$, namely crossvalidation (Ramsay and Silverman 2005), Akaike's information criterion (Akaike 1974), the bootstrap method (Hall and Vial 2006), the ratio method (Lam and Yao 2012) and explained variance (Chiou 2012). Here, the value of $K$ is determined as the minimum that reaches a certain level of the proportion of total variance explained by the $K$ leading components such that:

$$
K=\underset{K: K \geq 1}{\operatorname{argmin}}\left\{\sum_{k=1}^{K} \widehat{\lambda}_{k} / \sum_{k=1}^{\infty} \widehat{\lambda}_{k} \mathbb{1}_{\left\{\widehat{\lambda}_{k}>0\right\}} \geq P_{1}\right\},
$$

where $P_{1}=99 \%, \mathbb{1}_{\left\{\hat{\lambda}_{k}>0\right\}}$ is to exclude possible zero eigenvalues, and $\mathbb{1}\{\cdot\}$ represents the binary indicator function. When we align with Chantziara and Skiadopoulos (2008) and Cummins et al. (2016) and utilise three principal components, 99\% of the variation in our dataset is explained; therefore we also specify $P_{1}=99 \%$.

By characterising the relationship between oil futures prices of different expiries in the above manner, we obtain daily functional realisations of its term structure, or futures curve. With a set of realisations $\mathcal{X}(\theta)=\left\{\mathcal{X}_{1}(\theta), \ldots, \mathcal{X}_{n}(\theta)\right\}$, the mean function can be approximated as $\widehat{\mu}(\theta)=\sum_{t=1}^{n} \mathcal{X}_{t}(\theta) / n$, and the theoretical covariance function can be empirically estimated as:

$$
\widehat{c}_{n}=\frac{1}{n} \sum_{i=1}^{n}\left\langle\mathcal{X}_{t}, x\right\rangle \mathcal{X}_{t}, \quad x \in \mathcal{H},
$$

which is shown to be consistent under weak dependency (see, e.g., Hörmann and Kokoszka 2010). From the empirical covariance function, we can extract estimated functional principal component functions $\boldsymbol{B}=\left\{\widehat{\phi}_{1}(\theta), \ldots, \widehat{\phi}_{K}(\theta)\right\}$. Conditioning on the past functions $\mathcal{X}(\theta)$ and the estimated functional principal components $\boldsymbol{B}$, the one-step-ahead point forecast of $\mathcal{X}_{n+1}(\theta)$ can be expressed as:

$$
\begin{aligned}
\widehat{\mathcal{X}}_{n+1 \mid n}(\theta) & =\mathrm{E}\left[\mathcal{X}_{n+1}(\theta) \mid \boldsymbol{\mathcal { X }}(\theta), \boldsymbol{B}\right] \\
& =\widehat{\mu}(\theta)+\sum_{k=1}^{K} \widehat{\beta}_{n+1 \mid n, k} \widehat{\phi}_{k}(\theta),
\end{aligned}
$$

where $\widehat{\beta}_{n+1 \mid n, k}$ denotes time series forecasts of the $k^{\text {th }}$ component scores. The forecasts of these scores can be obtained via a univariate time series forecasting method, such as exponential smoothing (see Ord and Fildes 2013, for a review), which can handle non-stationarity of the functional principal component scores.

Following early work by Gardner, Jr and McKenzie (1985), we consider a damped trend exponential smoothing model, where a parameter $0 \leq \xi<1$ dampens the trend to a flat line sometime in the future. For each set of principal component scores, the resulting model is expressed 
as:

$$
\begin{aligned}
& \beta_{t}=\ell_{t-1}+\xi b_{t-1}+\epsilon_{t}, \\
& \ell_{t}=\ell_{t-1}+\xi b_{t-1}+\delta \epsilon_{t}, \\
& b_{t}=\xi b_{t-1}+\gamma \epsilon_{t},
\end{aligned}
$$

where $\ell_{t}$ denotes level at time $t=1, \ldots, n, b_{t}$ denotes the growth rate and $\epsilon_{t}$ denotes the error term. Elton et al. (2006) also consider exponential smoothing for financial economic forecasting.

\subsection{Benchmarks}

To evaluate the performance of our proposed FTS framework, we employ the use of fundamental and latent factor benchmarks. We utilise fundamental factors proposed by Andreasson et al. (2016) to model commodity futures prices, namely the Trade Weighted US Dollar Exchange Rate, S\&P 500 Index levels, the VIX (a measure of the market's expectation of future S\&P 500 index volatility) and the EcPol developed by Baker et al. (2015). The model is specified as follows:

$$
\mathrm{Oil}_{t}^{\tau}=\zeta+\beta_{1} \mathrm{SP}_{500}+\beta_{t-1} \mathrm{VIX}_{t-1}+\beta_{3} \mathrm{USD}_{t-1}+\beta_{4} \mathrm{EcPol}_{t-1}+\varepsilon_{t},
$$

with $\mathrm{Oil}_{t}^{\tau}$ denoting the $\log$ return of the oil futures with expiry $\tau$ at time $t, \zeta$ denoting the intercept term, SP500 $t_{-1}$ denoting S\&P $500 \log$ return at time $t-1$, VIX $_{t-1}$ denoting VIX volatility index $\log$ change at time $t-1$, USD denoting the trade-weighted US dollar index at time $t-1, \mathrm{EcPol}_{t-1}$ denoting US Economic Policy Uncertainty Index log change at time $t-1$ and $\varepsilon_{t}$ denoting the error term at time $t$. We hereafter refer to this model as Fundamental.

Our second benchmark is the latent factor model, as proposed by Chantziara and Skiadopoulos (2008). They use three discrete principal component extractions as factors for forecasting, as follows:

$$
\mathrm{Oil}_{t}^{\tau}=\zeta+\beta_{1} \mathrm{f}_{1, t-1}+\beta_{2} \mathrm{f}_{2, t-1}+\beta_{3} \mathrm{f}_{3, t-1}+v_{t},
$$

where $f_{1, t-1}, f_{2, t-1}$ and $f_{3, t-1}$ denote the first, second and third principal components calculated from the oil futures prices, $\beta_{1}, \beta_{2}$ and $\beta_{3}$ denote the coefficients and $v_{t}$ denotes the error term. We hereafter refer to this model as PC.

Finally, as a naïve benchmark, we also incorporate the random walk without drift into our in-sample analysis. The random walk without drift model can be expressed as:

$$
\mathrm{Oil}_{n+1}^{\tau}=\mathrm{Oil}_{n}^{\tau}+v_{n+1}
$$

where $v_{n+1}$ is a zero-mean error term that is uncorrelated to $\mathrm{Oil}_{n}^{\tau}$. The random walk without drift is also utilised in the out-of-sample performance evaluation to produce the Mean Absolute Scaled Error (MASE) as proposed by Hyndman and Koehler (2006). The MASE loss function is further detailed in the following sections. 


\subsection{Forecast Evaluation}

Out-of-sample forecasts are obtained using an expanding scheme. Each day an additional observation is added to an expanding training window with the models being re-estimated. We choose to expand the training set and re-estimate the model at each time step, daily, to incorporate all available up-to-date information into our forecast. ${ }^{8}$ Using our January 2009-December 2015 dataset, we statistically compare the model forecasts using a 500-day out-of-sample window, whereby the January 2009-December 2013 period is initially designated as the training window with the calculated forecasts being compared with the 500 unknown observations in the December 2013-December 2015 window. As an additional robustness check that controls for sensitivity to the specific out-of-sample period, 250-day and 750-day out-of-sample windows are also specified. We evaluate in-sample predictions using (where appropriate) a variety of performance measures: Mean Absolute Error (MAE), Mean Mixed Error Under (MME(U)), Mean Mixed Error Over $(\mathrm{MME}(\mathrm{O}))$, and Mean Correct Predictor of the Direction of Change (MCPDC).

\subsection{Loss Functions}

We assess the performance of the models using the following measures:

1) MAE is the average of the absolute differences between the prediction, $\widehat{\mathrm{Oil}}_{n+\vartheta \mid n+\vartheta-1}$, and the corresponding observation, $\operatorname{Oil}_{n+\vartheta}$. It measures the average error magnitude of the predictions, regardless of error direction and serves to aggregate errors into a single measure of predictive power:

$$
\begin{aligned}
\operatorname{MAE}^{\tau} & =\frac{1}{T} \sum_{\vartheta=1}^{T}\left|\mathrm{Oil}_{n+\vartheta}^{\tau}-\widehat{\mathrm{Oil}}_{n+\vartheta \mid n+\vartheta-1}^{\tau}\right|, \quad \tau=1, \ldots, 11, \\
\mathrm{MAE}_{\text {overall }} & =\frac{1}{11} \sum_{\tau=1}^{11} \operatorname{MAE}^{\tau},
\end{aligned}
$$

where $\mathrm{Oil}_{n+\vartheta}^{\tau}$ and $\widehat{\mathrm{Oil}}_{n+\vartheta \mid n+\vartheta-1}^{\tau}$ are the observed values and their predictions for a particular expiry and model, and $T$ denotes the number of observations in the forecasting period.

2) Mean error (ME) is the average of the differences between the prediction, $\widehat{\mathrm{Oil}}_{n+\vartheta \mid n+\vartheta-1}$, and the corresponding observation, $\operatorname{Oil}_{n+\vartheta}$. It measures the average error magnitude of the predictions, taking into account error direction, and serves to aggregate errors into a single

\footnotetext{
${ }^{8}$ Alternatively, a rolling window estimation approach that would iteratively discard earlier data points could be adopted. However, we proceed on the basis that all data points are relevant in the estimation stage with the exponential smoothing forecasts weighting the most recent data points more heavily when producing the forecasts.
} 
measure of predictive power:

$$
\begin{aligned}
\mathrm{ME}^{\tau} & =\frac{1}{T} \sum_{\vartheta=1}^{T}\left(\mathrm{Oil}_{n+\vartheta}^{\tau}-\widehat{\mathrm{Oil}}_{n+\vartheta \mid n+\vartheta-1}^{\tau}\right), \quad \tau=1, \ldots, 11, \\
\mathrm{ME}_{\text {overall }} & =\frac{1}{11} \sum_{\tau=1}^{11} \mathrm{ME}^{\tau},
\end{aligned}
$$

where $\mathrm{Oil}_{n+\vartheta}^{\tau}$ and $\widehat{\mathrm{Oil}}_{n+\vartheta \mid n+\vartheta-1}^{\tau}$ are the observed values and their predictions for a particular expiry and model, and $T$ denotes the number of observations in the forecasting period.

3) MASE utilises the error produced by an in-sample naïve random walk without drift to scale and compare the out-of-sample errors observed. It measures the error magnitude scaled by the magnitude of the in-sample error:

$$
q_{\vartheta}^{\tau}=\frac{\mathrm{Oil}_{n+\vartheta}^{\tau}-\widehat{\mathrm{Oil}}_{n+\vartheta \mid n+\vartheta-1}^{\tau}}{\frac{1}{n-1} \sum_{\varphi=2}^{n}\left|\mathrm{Oil}_{\varphi}-\mathrm{Oil}_{\varphi-1}\right|}, \quad \vartheta=1, \ldots, T .
$$

The error metric is independent of the scale of the data. A scaled error is less than one if it produces a better forecast than the average one-step, naïve forecast computed in-sample (Hyndman and Koehler 2006). The MASE is simply:

$$
\text { MASE }=\frac{1}{T \tau} \sum_{\vartheta=1}^{T} \sum_{\tau=1}^{11}\left|q_{\vartheta}^{\tau}\right|
$$

4) $\mathrm{MME}$ is an asymmetric loss function. $\mathrm{MME}(\mathrm{U})$ penalises under-predictions more heavily, while $\operatorname{MME}(\mathrm{O})$ penalises over-predictions more heavily. It is not a scoring rule in the conventional sense. However, it is useful in that it provides us with an indication of the tendency of a model to under- or over-predict. This is an important consideration for consumers of oil, as under-predictions of future oil prices may be of greater concern to those in need of future supply:

$$
\begin{aligned}
\operatorname{MME}^{\tau}(\mathrm{U}) & =\frac{1}{T}\left[\sum_{\psi=\eta_{1}^{\mathrm{O}}}^{\eta_{m}^{\mathrm{O}}}\left|\mathrm{Oil}_{n+\psi}^{\tau}-\widehat{\mathrm{Oil}}_{n+\psi \mid n+\psi-1}^{\tau}\right|+\sum_{\psi=\eta_{1}^{\mathrm{U}}}^{\eta_{m}^{\mathrm{U}}} \sqrt{\left|\mathrm{Oil}_{n+\psi}^{\tau}-\widehat{\mathrm{Oil}}_{n+\psi \mid n+\psi-1}^{\tau}\right|}\right], \\
\operatorname{MME}^{\tau}(\mathrm{O}) & =\frac{1}{T}\left[\sum_{\psi=\eta_{1}^{\mathrm{U}}}^{\eta_{m}^{\mathrm{U}}}\left|\mathrm{Oil}_{n+\psi}^{\tau}-\widehat{\mathrm{Oiil}}_{n+\psi \mid n+\psi-1}^{\tau}\right|+\sum_{\psi=\eta_{1}^{\mathrm{O}}}^{\eta_{m}^{\mathrm{O}}} \sqrt{\left|\mathrm{Oil}_{n+\psi}^{\tau}-\widehat{\mathrm{Oil}}_{n+\psi \mid n+\psi-1}^{\tau}\right|}\right], \\
\operatorname{MME}(\mathrm{U})_{\text {overall }} & =\frac{1}{11} \sum_{\tau=1}^{11} \operatorname{MME}^{\tau}(\mathrm{U}), \\
\operatorname{MME}(\mathrm{O})_{\text {overall }} & =\frac{1}{11} \sum_{\tau=1}^{11} \operatorname{MME}^{\tau}(\mathrm{O}),
\end{aligned}
$$

where $\eta_{m}^{\mathrm{U}}$ denotes the number of under-predictions and $\eta_{m}^{\mathrm{O}}$ denotes the number of overpredictions. $\left\{\eta_{1}^{\mathrm{O}}, \ldots, \eta_{m}^{\mathrm{O}}\right\}$ represents the indices of the over-predictions, and $\left\{\eta_{1}^{\mathrm{U}}, \ldots, \eta_{m}^{\mathrm{U}}\right\}$ represents the indices of the under-predictions. 
5) The MCPDC is the percentage of predictions for which the prediction, $\widehat{\mathrm{Oil}}_{n+\vartheta \mid n+\vartheta-1}$, has the same sign as the corresponding observation, $\mathrm{Oil}_{n+\vartheta}$. MCPDC measures how well the model can predict the direction of movement, regardless of error magnitude.

When evaluating out-of-sample forecasts, as opposed to in-sample predictions, we refer to the above measures as Mean Absolute Forecast Error (MAFE), Mean Forecast Error (MFE), Mean Absolute Scaled Forecast Error (MASFE), Mean Mixed Forecast Error Under (MMFE(U)), Mean Mixed Forecast Error Over (MMFE(O)), and Mean Correct Forecast of the Direction of Change (MCFDC), respectively.

\section{Empirical Results}

\subsection{In-sample modelling}

We briefly outline in-sample fit before drawing inferences using measures of out-of-sample performance. First, we outline the number of principal components used. In line with Chantziara and Skiadopoulos (2008) and Cummins et al. (2016) we specify three discrete principal components to model the oil futures curve. This results in $99 \%$ of the variance in the data being explained. Therefore, in the functional context, we also seek to explain $99 \%$ of the total variation, resulting in two functional principal components being adapted for the FTS model. Interestingly, a third functional principal component contributes less than $0.1 \%$ explained variation. We model the relationship between the curves using the FTS decomposition shown in Figure 3. The first functional principal component can be interpreted as characterising the linear relationship between the contracts, with the second component addressing the need to model the curvature dynamic. Regression results from fitting the benchmark fundamental and PC models are available upon request.

We now seek to establish how well the models fit in an in-sample modelling exercise. In-sample modelling results using $\mathrm{MAE}, \mathrm{MCPDC}, \mathrm{MME}(\mathrm{U})$ and $\mathrm{MME}(\mathrm{O})$ loss functions are presented in Table 2. Each row shows the calculated performance measure on an individual expiry basis with the Overall row presenting the loss function aggregated across all contracts. In a cross-model comparison of the absolute and asymmetric performance measures the results of the in-sample fit using our training set are broadly similar. More specifically we observe the same Overall MAE result of 0.0129 across the PC, FTS and Fundamental models. ${ }^{9}$ This indicates that despite being constructed using distinct approaches each model closely calibrates to the in-sample patterns present in the dataset. Furthermore, this is in line with Cummins et al. (2016) who find no discernible in-sample difference between the competing oil futures models. Interestingly, all

\footnotetext{
${ }^{9}$ As shown in Table 2 Overall in-sample $\mathrm{MME}(\mathrm{U}) /(\mathrm{O})$ levels are also similar at 0.0562/0.0583, 0.0569/0.0575 and 0.0562/0.0582, for the PC, FTS and Fundamental models, respectively.
} 
FTS fit

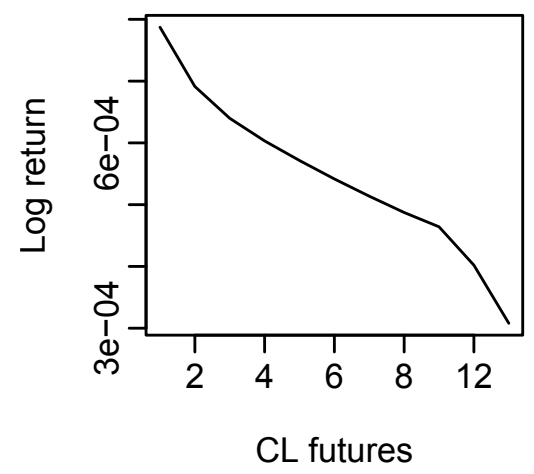

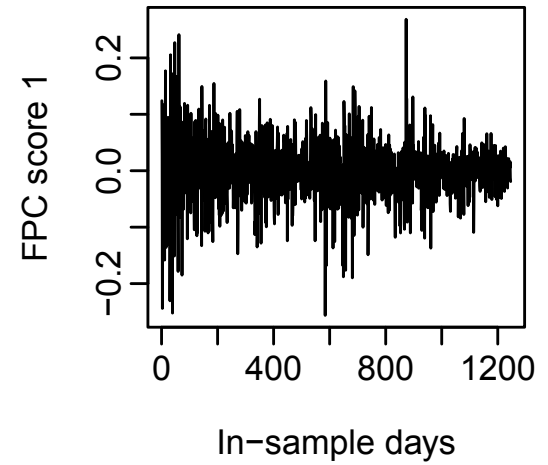

Decomposition
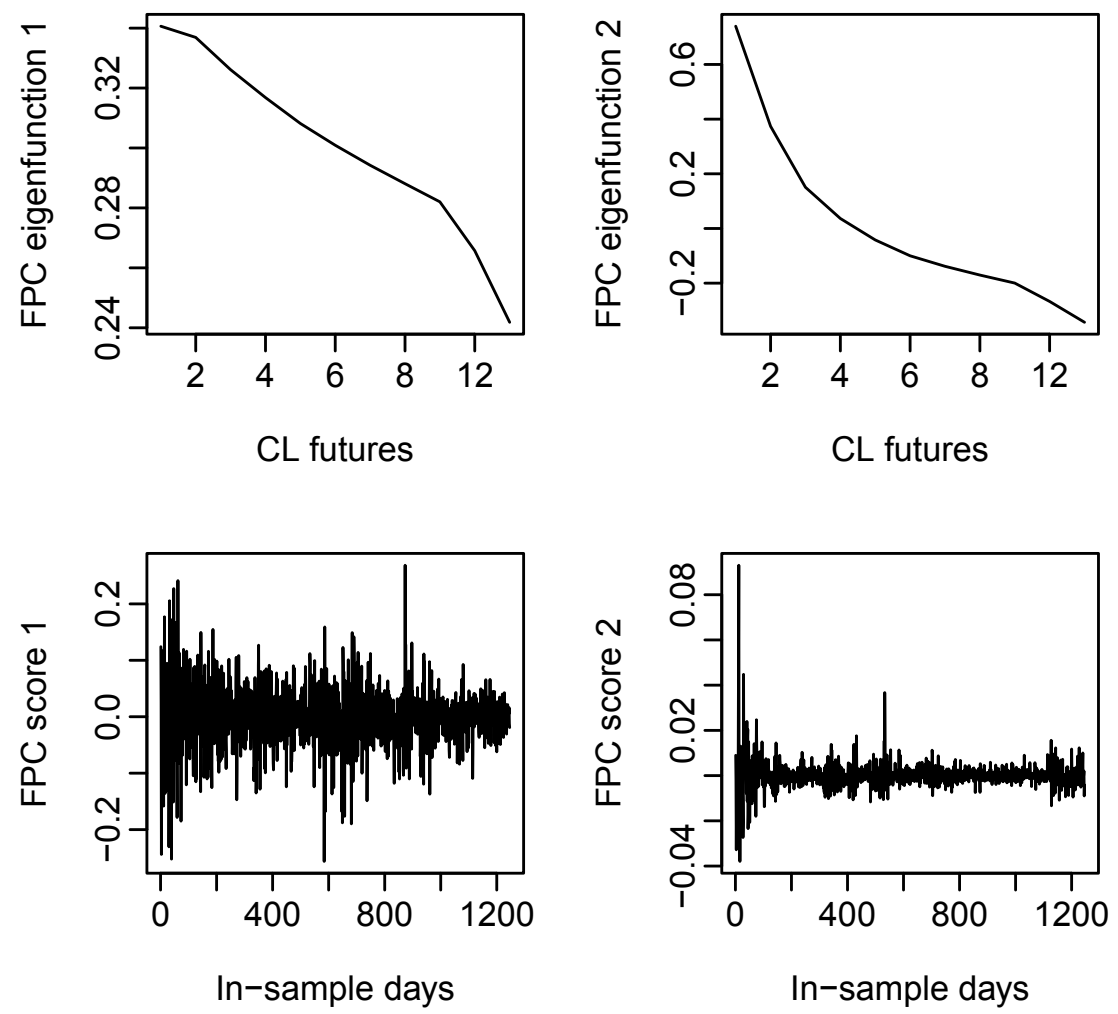

Figure 3: FTS in-sample fit: the mean fitted FTS (Functional Time Series) model and a graphical representation of its FPC (Functional Principal Component) decomposition over the January 2009-December 2013 period

models considered outperform the naïve random walk which led to an Overall MAE of 0.0188. Another notable result is that while all models correctly predict the direction of change better than would be achieved by chance alone (i.e., MCPDC> 0.5), the FTS boasts the greatest overall success, at $53.38 \%$, versus $52.70 \%$ for the Fundamental and $52.95 \%$ for the PC models.

\subsection{Out-of-sample forecasts}

To overcome the potential for in-sample data overfitting we derive inferences from our out-ofsample testing results. The out-of-sample framework aims to answer the question; how well do the models perform in terms of producing accurate forecasts of unknown future observations? We choose to employ a recursively expanding estimation window as it closely represents a situation a practitioner might find themselves in, whereby they use all available data up to today to forecast tomorrow's price. An alternative approach is to use a rolling fixed-length estimation window. We argue, however, that our expanding window approach represents a more appropriate environment for the exponential smoothing framework to determine its weightings, given that it internally deems observations in the distant past to have less of an effect on current forecasts.

Table 3 presents the loss functions produced under our out-of-sample exercise. To produce the dynamic iterative forecasts, the ftsa package of Hyndman and Shang (2018) was employed. The 


\begin{tabular}{|c|c|c|c|c|c|c|c|}
\hline \multirow[t]{2}{*}{ Expiry } & \multicolumn{4}{|c|}{ MAE } & \multicolumn{3}{|c|}{ MCPDC } \\
\hline & PC & FTS & Fund & RW & PC & FTS & Fund \\
\hline Overall & 0.0129 & 0.0129 & 0.0129 & 0.0188 & 0.5295 & 0.5338 & 0.5270 \\
\hline CL1 & 0.0152 & 0.0152 & 0.0152 & 0.0220 & 0.5325 & 0.5245 & 0.5277 \\
\hline CL2 & 0.0144 & 0.0145 & 0.0145 & 0.0209 & 0.5398 & 0.5213 & 0.5229 \\
\hline CL3 & 0.0139 & 0.0139 & 0.0139 & 0.0202 & 0.5261 & 0.5277 & 0.5205 \\
\hline CL4 & 0.0135 & 0.0135 & 0.0135 & 0.0197 & 0.5277 & 0.5285 & 0.5213 \\
\hline CL5 & 0.0131 & 0.0132 & 0.0131 & 0.0192 & 0.5301 & 0.5333 & 0.5237 \\
\hline CL6 & 0.0128 & 0.0128 & 0.0128 & 0.0187 & 0.5333 & 0.5357 & 0.5349 \\
\hline CL7 & 0.0125 & 0.0125 & 0.0125 & 0.0183 & 0.5285 & 0.5373 & 0.5333 \\
\hline CL8 & 0.0123 & 0.0123 & 0.0123 & 0.0180 & 0.5261 & 0.5365 & 0.5309 \\
\hline CL9 & 0.0120 & 0.0120 & 0.0120 & 0.0176 & 0.5253 & 0.5414 & 0.5317 \\
\hline CL12 & 0.0114 & 0.0114 & 0.0114 & 0.0167 & 0.5245 & 0.5414 & 0.5269 \\
\hline CL18 & 0.0105 & 0.0105 & 0.0105 & 0.0154 & 0.5309 & 0.5446 & 0.5229 \\
\hline \multirow[t]{2}{*}{ Expiry } & \multicolumn{3}{|c|}{ MME(U) } & & \multicolumn{3}{|c|}{$\mathrm{MME}(\mathrm{O})$} \\
\hline & PC & FTS & Fund & & PC & FTS & Fund \\
\hline Overall & 0.0562 & 0.0569 & 0.0562 & & 0.0583 & 0.0575 & 0.0582 \\
\hline CL1 & 0.0628 & 0.0637 & 0.0626 & & 0.0627 & 0.0618 & 0.0627 \\
\hline CL2 & 0.0608 & 0.0617 & 0.0609 & & 0.0615 & 0.0606 & 0.0615 \\
\hline CL3 & 0.0592 & 0.0600 & 0.0594 & & 0.0605 & 0.0597 & 0.0605 \\
\hline CL4 & 0.0581 & 0.0588 & 0.0583 & & 0.0596 & 0.0589 & 0.0596 \\
\hline CL5 & 0.0570 & 0.0577 & 0.0570 & & 0.0590 & 0.0583 & 0.0589 \\
\hline CL6 & 0.0560 & 0.0566 & 0.0560 & & 0.0583 & 0.0576 & 0.0582 \\
\hline CL7 & 0.0550 & 0.0558 & 0.0551 & & 0.0578 & 0.0571 & 0.0576 \\
\hline CL8 & 0.0543 & 0.0550 & 0.0543 & & 0.0573 & 0.0566 & 0.0571 \\
\hline CL9 & 0.0536 & 0.0543 & 0.0536 & & 0.0568 & 0.0561 & 0.0566 \\
\hline CL12 & 0.0519 & 0.0525 & 0.0519 & & 0.0552 & 0.0545 & 0.0550 \\
\hline CL18 & 0.0493 & 0.0498 & 0.0495 & & 0.0526 & 0.0519 & 0.0523 \\
\hline
\end{tabular}

Note: For each of the 11 different expiry continuous crude oil futures in our sample, this table presents the calculated performance measures (MAE, MME(U/O), and MCPDC) for the FTS, Fund (Fundamental) and PC models fitted to our daily dataset during the January 2009-December 2013 in-sample period. Overall refers to the loss function aggregated across all considered expiries. RW represents the random walk without drift. Because of its construction, it is not appropriate to calculate MCPDC and MME(U/O) for RW. 
FTS model shows a lower forecasted absolute error overall, with its forecasting improvement not being confined to any one segment of the futures curve. The MFE loss function also indicates that the FTS has the lowest error, both overall-at -0.0010 versus -0.0017 and -0.0016 , for FTS versus PC and Fundamental, respectively-and across each of the individual contract maturities. Furthermore, the use of the MASFE measure results in a similar conclusion with an error measure of 0.7286 overall for FTS versus 0.7324 and 0.7382 , for PC and Fundamental, respectively. When we focus on a purely directional loss function, the FTS model correctly predicts the direction of price change more often than PC and Fundamental, and, very importantly, more often than by chance alone (i.e., MCFDC > 0.5) for each expiry. Analysing the asymmetric $\operatorname{MMFE}(\mathrm{U})$ and $\operatorname{MMFE}(\mathrm{O})$ loss functions shows us that the FTS model has a comparative tendency to overestimate the price changeinformation that might be useful for instance, when adopting such forecasting frameworks for use in hedging or trading applications. ${ }^{10}$ The FTS model's comparative tendency to overestimate price changes might result in contrasting implications for those adopting it for hedging or speculative trading activities. ${ }^{11}$ Finally, using 250 and 750 days as alternative out-of-sample window lengths leads to qualitatively similar results being obtained.

Table 3: Out-of-sample Loss Functions

\begin{tabular}{lcccccc}
\hline Expiry & & MAFE & & \multicolumn{3}{c}{ MCFDC } \\
& PC & FTS & Fund & PC & FTS & Fund \\
\hline Overall & 0.0138 & $\mathbf{0 . 0 1 3 7}$ & 0.0139 & 0.5087 & $\mathbf{0 . 5 1 6 9}$ & 0.4831 \\
CL1 & $\mathbf{0 . 0 1 6 6}$ & $\mathbf{0 . 0 1 6 6}$ & 0.0168 & $\mathbf{0 . 5 4 8 0}$ & 0.5060 & 0.4640 \\
CL2 & $\mathbf{0 . 0 1 6 0}$ & $\mathbf{0 . 0 1 6 0}$ & 0.0162 & $\mathbf{0 . 5 4 4 0}$ & 0.5160 & 0.4640 \\
CL3 & 0.0154 & $\mathbf{0 . 0 1 5 3}$ & 0.0156 & 0.5020 & $\mathbf{0 . 5 1 8 0}$ & 0.4660 \\
CL4 & 0.0149 & $\mathbf{0 . 0 1 4 8}$ & 0.0151 & 0.4960 & $\mathbf{0 . 5 2 2 0}$ & 0.4820 \\
CL5 & 0.0144 & $\mathbf{0 . 0 1 4 3}$ & 0.0145 & 0.4960 & $\mathbf{0 . 5 2 2 0}$ & 0.4820 \\
CL6 & 0.0138 & $\mathbf{0 . 0 1 3 7}$ & 0.0139 & 0.4920 & $\mathbf{0 . 5 1 6 0}$ & 0.4860 \\
CL7 & 0.0135 & $\mathbf{0 . 0 1 3 4}$ & 0.0135 & 0.4960 & $\mathbf{0 . 5 2 8 0}$ & 0.4960 \\
CL8 & 0.0131 & $\mathbf{0 . 0 1 3 0}$ & 0.0131 & 0.5080 & $\mathbf{0 . 5 2 2 0}$ & 0.5020 \\
\hline
\end{tabular}

Continued on next page

\footnotetext{
${ }^{10}$ As shown in Table 3, Overall out-of-sample $\operatorname{MMFE}(\mathrm{U}) /(\mathrm{O})$ stand at $0.0646 / 0.0530,0.0624 / 0.0549$ and $0.0647 / 0.0536$, for PC, FTS and Fundamental, respectively.

${ }^{11}$ First, consider an airline as an example of a potential commercial hedger. With oil being an incurred operational cost, the use of a model that overestimates projected oil price changes, and hence increased volatility of future costs, might lead to management being too cautious in their business decisions. Second, consider an oil option trader as a potential speculative trader. If they were to adopt a model that overestimates underlying oil futures price changes, it could lead to them displaying too little caution and overpaying for the associated option. This is caused by price volatility in the underlying futures contract being a key option pricing component.
} 


\begin{tabular}{|c|c|c|c|c|c|c|}
\hline \multirow[t]{2}{*}{ Expiry } & \multicolumn{3}{|c|}{ MAFE } & \multicolumn{3}{|c|}{ MCFDC } \\
\hline & PC & FTS & Fund & PC & FTS & Fund \\
\hline CL9 & 0.0126 & 0.0125 & 0.0126 & 0.5020 & 0.5220 & 0.4880 \\
\hline CL12 & 0.0115 & 0.0115 & 0.0116 & 0.4960 & 0.5100 & 0.4920 \\
\hline CL18 & 0.0097 & 0.0096 & 0.0097 & 0.5160 & 0.5040 & 0.4920 \\
\hline \multirow[t]{2}{*}{ Expiry } & \multicolumn{3}{|c|}{ MASFE } & \multicolumn{3}{|c|}{ MFE } \\
\hline & PC & FTS & Fund & PC & FTS & Fund \\
\hline Overall & 0.7324 & 0.7286 & 0.7382 & -0.0017 & -0.0010 & -0.0016 \\
\hline CL1 & 0.7548 & 0.7551 & 0.7656 & -0.0021 & -0.0012 & -0.0021 \\
\hline CL2 & 0.7628 & 0.7627 & 0.7737 & -0.0019 & -0.0011 & -0.0019 \\
\hline CL3 & 0.7621 & 0.7587 & 0.7706 & -0.0018 & -0.0010 & -0.0018 \\
\hline CL4 & 0.7575 & 0.7526 & 0.7640 & -0.0018 & -0.0010 & -0.0017 \\
\hline CL5 & 0.7507 & 0.7452 & 0.7557 & -0.0017 & -0.0010 & -0.0017 \\
\hline CL6 & 0.7389 & 0.7335 & 0.7424 & -0.0017 & -0.0010 & -0.0016 \\
\hline CL7 & 0.7356 & 0.7290 & 0.7386 & -0.0016 & -0.0009 & -0.0016 \\
\hline CL8 & 0.7267 & 0.7220 & 0.7304 & -0.0016 & -0.0009 & -0.0015 \\
\hline CL9 & 0.7133 & 0.7077 & 0.7164 & -0.0015 & -0.0009 & -0.0015 \\
\hline CL12 & 0.6918 & 0.6873 & 0.6939 & -0.0014 & -0.0008 & -0.0014 \\
\hline CL18 & 0.6261 & 0.6228 & 0.6297 & -0.0013 & -0.0007 & -0.0012 \\
\hline \multirow[t]{2}{*}{ Expiry } & \multicolumn{3}{|c|}{ MMFE(U) } & \multicolumn{3}{|c|}{ MMFE(O) } \\
\hline & PC & FTS & Fund & PC & FTS & Fund \\
\hline Overall & 0.0646 & 0.0624 & 0.0647 & 0.0530 & 0.0549 & 0.0536 \\
\hline CL1 & 0.0728 & 0.0704 & 0.0733 & 0.0583 & 0.0608 & 0.0595 \\
\hline CL2 & 0.0710 & 0.0684 & 0.0711 & 0.0574 & 0.0598 & 0.0585 \\
\hline CL3 & 0.0694 & 0.0671 & 0.0698 & 0.0566 & 0.0584 & 0.0573 \\
\hline CL4 & 0.0682 & 0.0659 & 0.0684 & 0.0558 & 0.0574 & 0.0563 \\
\hline CL5 & 0.0667 & 0.0644 & 0.0668 & 0.0545 & 0.0561 & 0.0549 \\
\hline CL6 & 0.0649 & 0.0627 & 0.0648 & 0.0537 & 0.0555 & 0.0542 \\
\hline CL7 & 0.0643 & 0.0619 & 0.0641 & 0.0527 & 0.0541 & 0.0530 \\
\hline CL8 & 0.0628 & 0.0608 & 0.0627 & 0.0517 & 0.0533 & 0.0520 \\
\hline CL9 & 0.0610 & 0.0591 & 0.0612 & 0.0509 & 0.0523 & 0.0511 \\
\hline CL12 & 0.0579 & 0.0560 & 0.0578 & 0.0484 & 0.0502 & 0.0488 \\
\hline CL18 & 0.0521 & 0.0499 & 0.0518 & 0.0436 & 0.0458 & 0.0443 \\
\hline
\end{tabular}


Note: For each of the 11 different expiry continuous crude oil futures in our sample, this table presents the calculated forecast performance measures (MAFE, MASFE, MFE, MMFE(U/O) and MCFDC) for the outof-sample forecasts produced by our FTS, Fund (Fundamental) and PC frameworks during the December 2013-December 2015 period. Overall refers to the loss function aggregated across all 11 expiries. The lowest forecast errors are shown in bold.

As these measures are simply indicators of an improvement in performance, we need to establish if the FTS model is indeed statistically superior. To this end, we turn to the MCS results presented in Table 4, where model confidence sets are constructed at confidence levels of $75 \%$ and $90 \%$ in line with Hansen et al. (2011). Details of the MCS framework are presented in the Appendix. As also outlined there, absolute error is adapted to distinguish between models in terms of out-of-sample performance. From the Overall row, the FTS resides exclusively in the superior set of models across the full futures curve, and for both test statistics. Furthermore, when focusing on each of the individual futures expiries, the FTS is classified, although not always exclusively, as a superior model in each case. Our MCS approach robustly identifies model outperformance, with its design inherently controlling for multiple comparisons. Multiple comparisons is the issue that given a sufficient number of simultaneous tests seemingly significant outperformance will be uncovered by chance alone.

Table 5 presents the results from alternative forecast evaluation frameworks to show the robustness of our results to the choice of test selected. All of the pairwise tests of our proposed functional approach versus the Fundamental or PC models indicate that our FTS model outperforms at the 1\% significance level. This is consistent when employing the test of predictive accuracy in Diebold and Mariano (1995), the modified version in Harvey et al. (1997) and the test of conditional predictive ability in Giacomini and White (2006). When we focus on the collective tests that simultaneously evaluate all models with respect to a reference benchmark model, we again verify that the forecast produced by the FTS model is a significant improvement over those of the other models considered. More specifically, when we specify the PC model as a benchmark, the superiority is consistent at the $1 \%$ significance level for both White's(2000) reality check and Hansen's(2005) superior predictive ability tests. However, when we specify the Fundamental model as the benchmark, only the Hansen (2005) test is shown to be significant. This serves to highlight the increased power of the Hansen (2005) test over the White's(2000) reality check on which it is based. Overall, these results provide additional evidence to support the conclusion that the FTS forecasting approach is indeed significantly better than both the PC and Fundamental models. 


\begin{tabular}{|c|c|c|c|c|c|c|c|c|}
\hline \multirow[b]{2}{*}{ Statistic } & \multirow[b]{2}{*}{ Expiry } & \multicolumn{3}{|c|}{$\alpha=0.25$} & \multirow[b]{2}{*}{ Expiry } & \multicolumn{3}{|c|}{$\alpha=0.1$} \\
\hline & & PC & FTS & Fundamental & & PC & FTS & Fundamental \\
\hline \multirow[t]{12}{*}{$T_{\mathrm{R}, \mathrm{M}}$} & Overall & & $\dagger$ & & Overall & & $\ddagger$ & \multirow{24}{*}{$\ddagger$} \\
\hline & CL1 & + & + & & CL1 & $\ddagger$ & $\ddagger$ & \\
\hline & CL2 & + & + & & CL2 & $\ddagger$ & $\ddagger$ & \\
\hline & CL3 & + & + & & CL3 & $\ddagger$ & $\ddagger$ & \\
\hline & CL4 & & $t$ & & CL4 & $\ddagger$ & $\ddagger$ & \\
\hline & CL5 & & + & & CL5 & $\ddagger$ & $\ddagger$ & \\
\hline & CL6 & & $t$ & & CL6 & $\ddagger$ & $\ddagger$ & \\
\hline & CL7 & & + & & CL7 & & $\ddagger$ & \\
\hline & CL8 & & $t$ & & CL8 & $\ddagger$ & $\ddagger$ & \\
\hline & CL9 & & + & & CL9 & $\ddagger$ & $\ddagger$ & \\
\hline & CL12 & $\dagger$ & $\dagger$ & & CL12 & $\ddagger$ & $\ddagger$ & \\
\hline & CL18 & + & + & & CL18 & $\ddagger$ & $\ddagger$ & \\
\hline \multirow[t]{12}{*}{$T_{\max , \mathrm{M}}$} & Overall & & $\dagger$ & & Overall & & $\ddagger$ & \\
\hline & CL1 & $t$ & + & & CL1 & $\ddagger$ & $\ddagger$ & \\
\hline & CL2 & + & + & & CL2 & $\ddagger$ & $\ddagger$ & \\
\hline & CL3 & $\dagger$ & + & & CL3 & $\ddagger$ & $\ddagger$ & \\
\hline & CL4 & & + & & CL4 & $\ddagger$ & $\ddagger$ & \\
\hline & CL5 & & $t$ & & CL5 & $\ddagger$ & $\ddagger$ & \\
\hline & CL6 & & $t$ & & CL6 & $\ddagger$ & $\ddagger$ & \\
\hline & CL7 & & + & & CL6 & $\ddagger$ & $\ddagger$ & \\
\hline & CL8 & & + & & CL8 & $\ddagger$ & $\ddagger$ & \\
\hline & CL9 & & $t$ & & CL9 & $\ddagger$ & $\ddagger$ & \\
\hline & CL12 & + & + & & CL12 & $\ddagger$ & $\ddagger$ & \\
\hline & CL18 & $\dagger$ & + & & CL18 & $\ddagger$ & $\ddagger$ & \\
\hline
\end{tabular}

Note: For each of the 11 different expiry continuous crude oil futures in our sample, this table presents the results of the model confidence test of Hansen et al. (2011) based on the out-of-sample forecasts produced by PC, our FTS and Fundamental methods during the December 2013-December 2015 period. Overall refers to forecasts aggregated across each of the 11 expiries: $\dagger$ and $\ddagger$ are used to indicate that the framework resides in the superior set of models at $75 \%$ and $90 \%$ confidence levels, respectively. 
Table 5: Alternative Statistical Tests

\begin{tabular}{lcccc}
\hline & \multicolumn{2}{c}{ Pairwise Tests } & \multicolumn{2}{c}{ Collective Tests } \\
\cline { 2 - 5 } Test & FTS Vs Fund & FTS Vs PC & Fund Benchmark PC Benchmark \\
\hline Diebold-Mariano (1995) & $-0.0038^{* * *}$ & $-0.0096^{* * *}$ & & \\
& $(0.0030)$ & $(0.0077)$ & & \\
Modified Diebold-Mariano (1997) & $-8.8250^{* * *}$ & $-3.0326^{* * *}$ & & \\
& $(0.0000)$ & $(0.0012)$ & 0.0000 & $(0.0000)$ \\
White (2000) Reality Check & & & $(0.6910)$ & $0.1118^{* * *}$ \\
Hansen (2005) SPA & & & $0.6365^{* * *}$ & $(0.0000)$ \\
Giacomini-White (2006) CPA & $76.8000^{* * *}$ & $10.4300^{* * *}$ & $(0.0000)$ & \\
\hline
\end{tabular}

Note: This table presents alternative tests for statistical outperformance across all the contracts considered; $p$-values associated with each test are given in parentheses beneath the calculated test statistics. ${ }^{*},{ }^{* *}, * * *$, represent significance at the $10 \%, 5 \%$, and $1 \%$ levels, respectively. The models considered are FTS, PC and Fund (Fundamental); CPA, conditional predictive ability; SPA, superior predictive ability. Please see underlying texts indicated for further technical implementation details. 


\section{Concluding Remarks}

Despite a strong practitioner need to model and accurately forecast oil futures prices, recent academic research has been unable to establish a discernible difference between the performance of competing models. We contribute to this strand of literature through the innovative introduction of an infinite dimensional class of factors, based on exponential smoothing forecasts of functional PCs. Our functional approach has many advantages. First, it circumvents the need to explicitly identify factors that model the shape of the oil futures curve. Second, it takes advantage of information that traditional models overlook; despite only certain contracts being actively quoted and traded on any given day, there is an underlying continuous smooth process linking oil futures contracts of different expiries. Finally, it is flexible in the sense that it is data driven, leaving the potential for it to be extended to other applications.

Our focus on WTI crude oil futures is motivated by the critical role of derivatives trading in Europe, as highlighted by Carroll et al. (2017), and the growing importance of WTI crude in a European context. We present out-of-sample results that provide empirical support for the adoption of our FTS approach when forecasting WTI oil futures, with the functional model residing in the superior set of models for all considered expiries. We now outline how our results link with previous studies.

First, our in-sample results agree with Cummins et al. (2016), who highlight that an in-sample fitting indicates no discernible difference between latent and macroeconomic factors when modelling oil futures. However, our proposal to employ the FTS model in the out-of-sample context contradicts their conclusions by uncovering a statistically outperforming forecasting model. Second, the contrasting in- and out-of-sample findings suggest that instability or overfitting might be influencing forecasting evaluation. These statistical phenomena have previously been highlighted by Giacomini and Rossi (2009) and Clark (2004), respectively. Third, our results also highlight that moving beyond the discrete approaches of Chantziara and Skiadopoulos (2008) and Andreasson et al. (2016) into a continuous FTS domain yields strong empirical benefits. As well as the importance of oil price forecasts for decision making as outlined in Section 1, our uncovered forecasting outperformance could also inform economically exploitable speculative trading or hedging strategies, with their success being dependent on an individual investor's trading costs. Future work in this area might consider alternative benchmark models and forecasting horizons, seek to construct a semi-parametric model by supplementing our FTS framework with fundamental exogenous factors, or further refine forecast accuracy through a forecast combination approach such as employed by Gao and Nardari (2018). 


\section{Acknowledgments}

The authors are grateful for the insightful comments and suggestions of the participants at the Irish Statistical Association Workshop on Frontiers in Functional Data Analysis and the Forecasting Financial Markets Conference. Han Lin Shang acknowledges the financial support of a research grant from the College of Business and Economics at the Australian National University. 


\section{References}

Akaike, H. (1974), 'A new look at the statistical model identification', IEEE Transactions on Automatic Control 19(6), 716-723.

Alquist, R., Kilian, L. and Vigfusson, R. J. (2013), Forecasting the price of oil, in G. Elliott, C. Granger and A. Timmermann, eds, 'Handbook of Economic Forecasting', Vol. 2, North-Holland: Amsterdam, The Netherlands, chapter 8, pp. 427-507.

Andreasson, P., Bekiros, S., Nguyen, D. K. and Uddin, G. S. (2016), 'Impact of speculation and economic uncertainty on commodity markets', International Review of Financial Analysis 43, 115127.

Baker, S. R., Bloom, N. and Davis, S. J. (2015), Measuring economic policy uncertainty, Working paper 21633, National Bureau of Economic Research.

URL: http://www.policyuncertainty.com/media/BakerBloomDavis.pdf

Baruník, J. and Malinská, B. (2016), 'Forecasting the term structure of crude oil futures prices with neural networks', Applied Energy 164, 366-379.

Basak, S. and Pavlova, A. (2016), 'A model of financialization of commodities', The Journal of Finance 71(4), 1511-1556.

Bernardi, M. and Catania, L. (2015), The model confidence set package for R, Working paper 362, University of Rome "Tor Vergata".

URL: ftp://www . ceistorvergata.it/repec/rpaper/RP362.pdf

Bernardi, S., Leippold, M. and Lohre, H. (2018), 'Maximum diversification strategies along commodity risk factors', European Financial Management 24(1), 53-78.

Carroll, A., O'Brien, F. and Ryan, J. (2017), 'An examination of European firms derivatives usage: the importance of model selection', European Financial Management 23(4), 648-690.

Casassus, J. and Collin-Dufresne, P. (2005), 'Stochastic convenience yield implied from commodity futures and interest rates', The Journal of Finance 60(5), 2283-2331.

Casassus, J., Liu, P. and Tang, K. (2013), ‘Economic linkages, relative scarcity, and commodity futures returns', Review of Financial Studies 26(5), 1324-1362.

Chantziara, T. and Skiadopoulos, G. (2008), 'Can the dynamics of the term structure of petroleum futures be forecasted? Evidence from major markets', Energy Economics 30(3), 962-985. 
Chen, Y. and Li, B. (2017), 'An adaptive functional autoregressive forecast model to predict electricity price curves', Journal of Business \& Economic Statistics 35(3), 371-388.

Chiou, J.-M. (2012), ‘Dynamical functional prediction and classification with application to traffic flow prediction', The Annals of Applied Statistics 6(4), 1588-1614.

Clark, T. E. (2004), 'Can out-of-sample forecast comparisons help prevent overfitting?', Journal of forecasting 23(2), 115-139.

Cummins, M., Dowling, M. and Kearney, F. (2016), ‘Oil market modelling: A comparative analysis of fundamental and latent factor approaches', International Review of Financial Analysis 46, 211218.

Diebold, F. X. and Mariano, R. S. (1995), 'Comparing predictive accuracy', Journal of Business \& Economic Statistics pp. 253-263.

Elton, E. J., Gruber, M. J. and Spitzer, J. (2006), 'Improved estimates of correlation coefficients and their impact on optimum portfolios', European Financial Management 12(3), 303-318.

Engle, R. F. (2000), 'The econometrics of ultra-high-frequency data', Econometrica 68(1), 1-22.

Gao, X. and Nardari, F. (2018), 'Do commodities add economic value in asset allocation? new evidence from time-varying moments', Journal of Financial and Quantitative Analysis 53(1), 365393.

Gardner, Jr, E. S. and McKenzie, E. (1985), 'Forecasting trends in time series', Management Science 31(10), 1237-1246.

Giacomini, R. and Rossi, B. (2009), 'Detecting and predicting forecast breakdowns', The Review of Economic Studies 76(2), 669-705.

Giacomini, R. and White, H. (2006), 'Tests of conditional predictive ability', Econometrica 74(6), 1545-1578.

Hall, P. and Vial, C. (2006), 'Assessing the finite dimensionality of functional data', Journal of the Royal Statistical Society (Series B) 68(4), 689-705.

Hansen, P. R. (2005), 'A test for superior predictive ability', Journal of Business \& Economic Statistics 23(4), 365-380.

Hansen, P. R., Lunde, A. and Nason, J. M. (2011), 'The model confidence set', Econometrica 79(2), 453-497. 
Harvey, D., Leybourne, S. and Newbold, P. (1997), 'Testing the equality of prediction mean squared errors', International Journal of Forecasting 13(2), 281-291.

Hörmann, S. and Kokoszka, P. (2010), 'Weakly dependent functional data', The Annals of Statistics 38(3), 1845-1884.

Horváth, L., Kokoszka, P. and Rice, G. (2014), 'Testing stationarity of functional time series', Journal of Econometrics 179(1), 66-82.

Horváth, L. and Rice, G. (2015), 'Testing for independence between functional time series', Journal of Econometrics 189(2), 371-382.

Hyndman, R. J. and Koehler, A. B. (2006), 'Another look at measures of forecast accuracy', International Journal of Forecasting 22(4), $679-688$.

Hyndman, R. J. and Shang, H. L. (2018), ftsa: Functional Time Series Analysis. R package version 5.2.

URL: https : //CRAN.R-project.org/package=ftsa

Hyndman, R., Koehler, A. B., Ord, J. K. and Snyder, R. D. (2008), Forecasting with Exponential Smoothing: the State Space Approach, Springer Science \& Business Media, Heidelberg.

Kearney, F., Cummins, M. and Murphy, F. (2018), 'Forecasting implied volatility in foreign exchange markets: A functional time series approach', The European Journal of Finance 24(1), 1-18.

Kearney, F., Murphy, F. and Cummins, M. (2015), 'An analysis of implied volatility jump dynamics: Novel functional data representation in crude oil markets', The North American Journal of Economics and Finance 33, 199-216.

Kilian, L. (2009), 'Not all oil price shocks are alike: Disentangling demand and supply shocks in the crude oil market', American Economic Review 99(3), 1053-1069.

Kokoszka, P., Miao, H., Reimherr, M. and Taoufik, B. (2018), 'Dynamic functional regression with application to the cross-section of returns', Journal of Financial Econometrics 16(3), 461-485.

Kokoszka, P., Miao, H. and Zhang, X. (2015), 'Functional dynamic factor model for intraday price curves', Journal of Financial Econometrics 13(2), 456-477.

Kowal, D. R., Matteson, D. S. and Ruppert, D. (2017), 'A Bayesian multivariate functional dynamic linear model', Journal of the American Statistical Association 112(518), 733-744. 
Kowal, D. R., Matteson, D. S. and Ruppert, D. (2018), 'Functional autoregression for sparsely sampled data', Journal of Business \& Economic Statistics forthcoming.

Kyriakou, I., Nomikos, N. K., Papapostolou, N. C. and Pouliasis, P. K. (2016), 'Affine-structure models and the pricing of energy commodity derivatives', European Financial Management 22(5), 853-881.

Lam, C. and Yao, Q. (2012), 'Factor modelling for high-dimensional time series: Inference for the number of ffactor', The Annals of Statistics 40(2), 694-726.

Laukaitis, A. (2008), 'Functional data analysis for cash flow and transactions intensity continuous-time prediction using Hilbert-valued autoregressive processes', European Journal of Operational Research 185(3), 1607-1614.

Laws, J. and Thompson, J. (2004), 'The efficiency of financial futures markets: Tests of prediction accuracy', European Journal of Operational Research 155(2), 284-298.

Liu, X., Xiao, H. and Chen, R. (2016), 'Convolutional autoregressive models for functional time series', Journal of Econometrics 194(2), 263-282.

Müller, H.-G., Sen, R. and Stadtmüller, U. (2011), 'Functional data analysis for volatility', Journal of Econometrics 165(2), 233-245.

Neumann, M. and Skiadopoulos, G. (2013), 'Predictable dynamics in higher-order risk-neutral moments: Evidence from the s\&p 500 options', Journal of Financial and Quantitative Analysis 48(3), 947-977.

Ng, V. K. and Pirrong, S. C. (1996), 'Price dynamics in refined petroleum spot and futures markets', Journal of Empirical Finance 2(4), 359 - 388.

Ord, K. and Fildes, R. (2013), Principles of Business Forecasting, Cengage Learning, Mason, OH, USA.

Ramsay, J. and Silverman, B. (2005), Functional Data Analysis, 2nd edn, Springer Series in Statistics, New York.

Schwartz, E. and Smith, J. E. (2000), 'Short-term variations and long-term dynamics in commodity prices', Management Science 46(7), 893-911.

Sévi, B. (2014), 'Forecasting the volatility of crude oil futures using intraday data', European Journal of Operational Research 235(3), 643-659. 
Singleton, K. J. (2013), 'Investor flows and the 2008 boom/bust in oil prices', Management Science 60(2), 300-318.

Taylor, J. W. (2010), 'Triple seasonal methods for short-term electricity demand forecasting', European Journal of Operational Research 204(1), 139-152.

Trolle, A. B. and Schwartz, E. S. (2009), 'Unspanned stochastic volatility and the pricing of commodity derivatives', Review of Financial Studies 22(11), 4423-4461.

Tsay, R. S. (2016), 'Some methods for analyzing big dependent data', Journal of Business \& Economic Statistics 34(4), 673-688.

Wang, S., Jank, W. and Shmueli, G. (2008), 'Explaining and forecasting online auction prices and their dynamics using functional data analysis', Journal of Business \& Economic Statistics 26(2), 144-160.

White, H. (2000), 'A reality check for data snooping', Econometrica 68(5), 1097-1126.

Zhang, X. (2016), 'White noise testing and model diagnostic checking for functional time series', Journal of Econometrics 194(1), 76-95. 


\section{Appendix A: Model Confidence Set}

To examine statistical significance among the three methods, we consider the Model Confidence Set (MCS). The MCS procedure proposed by Hansen et al. (2011) consists of a sequence of tests which permits to construct a set of "superior" model, where the null hypothesis of equal predictive ability (EPA) is not rejected at a certain confidence level. As the EPA test statistic can be evaluated for any loss function, we adopt the tractable absolute error measure.

The procedure begins with an initial set of models of dimension $m=3$ encompassing all the model considered, $M_{0}=\left\{M_{1}, M_{2}, M_{3}\right\}$. For a given confidence level, a smaller set, the superior set of models $\widehat{M}_{1-\alpha}^{*}$ is determined where $m^{*} \leq m$. The best scenario is when the final set consists of a single model, i. e., $m=1$. Let $d_{i j, t}$ denotes the loss differential between two models $i$ and $j$, that is

$$
d_{i j, t}=l_{i, t}-l_{j, t}, \quad i, j=1, \ldots, m, \quad t=1, \ldots, n,
$$

and calculate

$$
d_{i, t}=\frac{1}{m-1} \sum_{j \in \mathrm{M}} d_{i j, t}, \quad i=1, \ldots, m
$$

as the loss of model $i$ relative to any other model $j$ at time point $t$. The EPA hypothesis for a given set of $\mathrm{M}$ candidate models can be formulated in two ways:

$$
\begin{array}{ll}
\mathrm{H}_{0, \mathrm{M}}: c_{i j}=0, & \text { for all } i, j=1,2, \ldots, m \\
\mathrm{H}_{\mathrm{A}, \mathrm{M}}: c_{i j} \neq 0, & \text { for some } i, j=1,2, \ldots, m .
\end{array}
$$

or

$$
\begin{array}{ll}
\mathrm{H}_{0, \mathrm{M}}: c_{i .}=0, & \text { for all } \quad i, j=1,2, \ldots, m \\
\mathrm{H}_{\mathrm{A}, \mathrm{M}}: c_{i .} \neq 0, \quad \text { for some } \quad i, j=1,2, \ldots, m
\end{array}
$$

where $c_{i j}=\mathrm{E}\left(d_{i j}\right)$ and $c_{i}=\mathrm{E}\left(d_{i}\right)$ are assumed to be finite and time independent. Based on $c_{i j}$ or

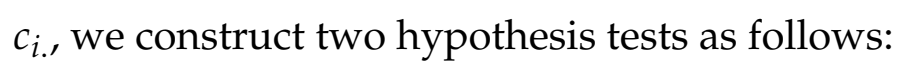

$$
t_{i j}=\frac{\bar{d}_{i j}}{\sqrt{\widehat{\operatorname{Var}}\left(\bar{d}_{i j}\right)}}, \quad t_{i .}=\frac{\bar{d}_{i .}}{\sqrt{\widehat{\operatorname{Var}}\left(\bar{d}_{i .}\right)}},
$$

where $\bar{d}_{i .}=\frac{1}{m-1} \sum_{j \in \mathrm{M}} \bar{d}_{i j}$ is the sample loss of $i^{\text {th }}$ model compared to the averaged loss across models in the set $M$, and $\bar{d}_{i j}=\frac{1}{n} \sum_{t=1}^{n} d_{i j, t}$ measures the relative sample loss between the $i^{\text {th }}$ and $j^{\text {th }}$ models. Note that $\widehat{\operatorname{Var}}\left(\bar{d}_{i}\right)$ and $\widehat{\operatorname{Var}}\left(\bar{d}_{i j}\right)$ are the bootstrapped estimates of $\operatorname{Var}\left(\bar{d}_{i}\right)$ and $\operatorname{Var}\left(\bar{d}_{i j}\right)$, respectively. According to Hansen et al. (2011) and Bernardi and Catania (2015), we perform a block bootstrap procedure with 5,000 bootstrap samples, where the block length $p$ is 
given by the maximum number of significant parameters obtained by fitting an $\operatorname{AR}(p)$ process on all the $d_{i j}$ term. For both hypotheses in (A.1) and (A.2), there exist two test statistics:

$$
T_{\mathrm{R}, \mathrm{M}}=\max _{i, j \in \mathrm{M}}\left|t_{i j}\right|, \quad T_{\max , \mathrm{M}}=\max _{i \in \mathrm{M}} t_{i}
$$

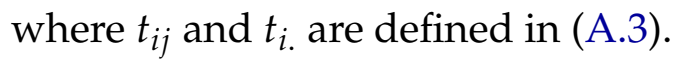

The MCS procedure is a sequential testing procedure, which eliminates the worst model at each step until the hypothesis of equal predictive ability is accepted for all the models belonging to a set of superior models. The selection of the worst model is determined by an elimination rule that is consistent with the test statistic,

$$
e_{\mathrm{R}, \mathrm{M}}=\underset{i \in M}{\arg \max }\left\{\sup _{j \in M} \frac{\bar{d}_{i j}}{\sqrt{\widehat{\operatorname{Var}}\left(\bar{d}_{i j}\right)}}\right\}, \quad e_{\max , \mathrm{M}}=\underset{i \in M}{\arg \max } \frac{\bar{d}_{i .}}{\widehat{\operatorname{Var}}\left(\bar{d}_{i .}\right)} .
$$

To summarise, the MCS procedure to obtain a superior set of models consists of the following steps:

1) Set $M=M_{0}$;

2) If the null hypothesis is accepted, then $M^{*}=M$; otherwise use the elimination rules defined in (A.4) to determine the worst model;

3) Remove the worst model, and go to Step 2). 